Voix et Images

voixetimages

\title{
La grammaire amoureuse de Ducharme
}

\section{Michel Biron}

Volume 25, numéro 2 (74), hiver 2000

Le champ littéraire de la jeunesse au carrefour de la recherche universitaire

URI : https://id.erudit.org/iderudit/201486ar

DOI : https://doi.org/10.7202/201486ar

Aller au sommaire du numéro

Éditeur(s)

Université du Québec à Montréal

ISSN

0318-9201 (imprimé)

1705-933X (numérique)

Découvrir la revue

Citer cet article

Biron, M. (2000). La grammaire amoureuse de Ducharme. Voix et Images, 25(2), 377-383. https://doi.org/10.7202/201486ar d'utilisation que vous pouvez consulter en ligne.

https://apropos.erudit.org/fr/usagers/politique-dutilisation/ 


\title{
Roman
}

\section{La grammaire amoureuse de Ducharme}

\author{
Michel Biron, Université du Québec à Montréal
}

Admirable continuité de Ducharme, qui prolonge avec Gros mots $^{1}$ une ouvre romanesque dont je ne vois pas l'équivalent dans la littérature québécoise. Mais peut-être at-on oublié ce que c'est, une ouvre? Le mot, honni pendant longtemps par la critique littéraire moderne, a un parfum suranné qui surprend à plus forte raison à propos d'une écriture où rien vraiment ne s'achève. Je n'en vois pas d'autres toutefois pour parler de cet ensemble de textes qui, tout différents qu'ils sont les uns par rapport aux autres, sont reconnaissables entre tous. L'œuvre s'oppose à l'esquisse et le travail qu'elle exige, à la tristesse de l'improvisation; elle suppose une intensité durable, qui n'est pas seulement du désir, mais de la volonté, de l'obstination, de la nervosité patiente. Chef-d'œuvre? C'est autre chose, qui ne correspond, je crois, à aucun des romans de Ducharme, ce neuvième encore moins que les précédents.

Quelle que soit la volonté qu'il mette à rester en piste, Johnny, le narrateur de Gros mots, se sent menacé d'expulsion. "C'est mon histoire, affirme-t-il dès le début. On est ici chez moi. On ne va pas me déloger comme ça. Se débarrasser du héros en trois coups de cuiller à mots." (p. 9) Ne veut-il pas se révolter, détruire les autres ou, mieux encore, s'autodétruire, se "branle-basser", comme on disait au temps du Nez qui voque ${ }^{2}$ ? Non, il n'est ni vraiment révolté ni suicidaire. Seul l'amour l'intéresse, le sacré comme le grossier, le pur comme le sexuel, l'invécu comme l'invivable. Cela ne va pas de soi dans un monde qui menace d'éliminer le héros pour trois fois rien, pour peu qu'il fléchisse un instant et qu'il réduise l'intensité avec laquelle il s'acharne à parcourir les mêmes lieux, à fréquenter les mêmes milieux, à tenir le même langage. Johnny, on l'apprend peu à peu à travers les personnages qui l'entourent, vient de Lavaltrie, il est dans la trentaine, il ne travaille pas, il habite une île près de Montréal, au crochet de la furieuse Exa Torrent, il aime comme une sœur - ce qui, chez Ducharme, signifie: de manière absolue - une autre femme, appelée Petite Tare, celle-ci est l'épouse de son frère adoptif Julien, il fréquente un bar où travaille Poppée. L'habituel, quoi. La société de Johnny ressemble à celle de tout héros ducharmien.

S'y ajoute un intrus, personnage neuf, le double plutôt que l'ami de Johnny. Il le découvre, par hasard, dans la jungle hivernale de son île, à l'intérieur d'un "cahier de vrais mots" trouvé dans les broussailles, dont il fera aussitôt une copie pour Petite Tare afin qu'elle le retranscrive au propre. Ensemble, ils glosent sur 
l'identité de l'auteur de ce cahier abandonné: "Les trávaux et les jours, on dirait, d'un impossible auteur, un complexé des grandeurs, un épris dont on n'a pas cru les cris trop forts en métaphores... Et la première page, et c'est ce qui m'a rachevé, est datée d'aujourd'hui, sans préciser l'année." (p. 18) Alter Ego ou Walter, c'est ainsi qu'ils désigneront plus loin l' impossible auteur" du cahier, creuse le roman de Ducharme en son centre et devient une sorte de personnage fétiche que l'on interprète comme on peut, c'est-à-dire avec le sentiment persistant que ce n'est jamais tout à fait ça.

Que lit-on dans ce cahier de Walter (bonjour au passage à André Gide)? L'Alter Ego aime Too Much, mais il a aussi sa "Petite Tare", appelée Bri, qui vit avec un autre Julien, nommé Ernie. Les personnages du cahier correspondent apparemment à ceux de l'histoire de Johnny, encore que les sentiments ne se distribuent pas de manière parfaitement symétrique. Pourquoi ce jeu approximatif de mise en abyme? Le titre même du roman le dit bien: les mots, gros ou petits, constituent l'horizon de tous les personnages, en particulier de Petite Tare, dont c'est la spécialité (elle a une maîtrise en lettres et projette de faire un "mémoire de doctorat "sur ce drôle d' "objet littéraire") (p. 295). Johnny et elle ponctuent leur lecture de remarques sur l'écriture de Walter, laquelle imite, en l'aggravant si possible, celle de Ducharme. En affirmant par exemple que le cahier du "pauvre Walter " n'a rien de littéraire, simple "trésor" personnel (p. 88), cahier de mots vrais, de paroles plutôt que de mots d'écrivain, le narrateur reprend l'un des leitmotive de Ducharme, à savoir son refus d'être considéré comme un "homme de lettres ${ }^{3}$ ". Raturé, malpropre, encombré de mauvais jeux de mots et d'idiosyncrasies, le cahier de Walter ressemble comme deux gouttes d'eau aux cahiers de Ducharme qu'on connait ${ }^{4}$. Petite Tare est plus sévère encore:
Walter a de la culture. À moins qu'il ne soit un écumeur de dictionnaire. Il n'a pas l'air. Il me fait plutôt l'effet d'un de ces imposteurs comme on en voit parmi les poètes. Un peu trop artistes à leur propre goût, exclus, ils se déguisent en pauvres types, ils font comme les autres. Ils le font si bien qu'on y croit, on les met dans le même tas. On leur réfléchit le personnage et ils se ramassent complètement piégés dedans. Ils n'en sortent plus. Ils ne peuvent plus... (p. 124)

Comme on le voit, Ducharme ne s'embarrasse pas de camoufler les clefs de lecture: il les donne au lecteur et s'assure plusieurs fois que celui-ci les a bien en main, ironisant plaisamment sur son écriture comme si celle-ci était, au fond, le sujet principal, voire unique de ce roman autoréflexif.

Qu'a-t-il de si extraordinaire, ce cahier de paroles? Simple confusion des noms, serait-on tenté de dire en éprouvant les mêmes sentiments que la pauvre Poppée lorsque Johnny lui fait toute une histoire pour lui cacher le nom de Petite Tare. "Are you nobody too", disait Emily Dickinson, citée (moins les tirets et les majuscules) par Johnny? (p. 12) Allez savoir. Walter est, au choix, un poète, un imposteur, un fou (il aurait appartenu à la huitième section des G.I's, celle des réformés pour 
désordres mentaux), Odilon Sansfaçon, O.S.F., finalement un voisin de l'autre île. Aucune importance d'ailleurs, le cahier est un leurre et on se demande même si le romancier ne s'est pas laissé emporter par son procédé, s'il n'y a pas cru trop fort. Piégé, Johnny ne parvient pas à détacher son regard du piège luimême, c'est-à-dire de son propre personnage. Autour de lui, les autres manquent d'espace pour se déployer: ni Petite Tare, ni Exa, malgré ses tentatives de suicide et son autorité domestique, ni la plantureuse Poppée, ni le fidèle Julien, avec qui Johnny se rend au Forum (nous sommes dans les années soixantedix, à l'époque, combien lointaine, où le Canadien gagnait plusieurs coupes Stanley de suite), ne font le poids à côté du moi envahissant de Johnny. Fasciné par son double, le héros se dissocie de lui-même comme il l'écrit avec des mots empruntés, en partie, au poème "Accompagnement " de Saint-Denys Garneau ${ }^{5}$ :

Walter me détache de moi de plus
en plus: de la même façon qu'en
le lisant je me mets au-dessus de
lui (sujets, hypostase), je me mets
derrière moi en marchant et je me
regarde aller, comme un idiot, ou
deux idiots, ou toute une filée,
l'un réfléchissant l'autre à
répétition, en chenilles proces-
sionnaires. (p. 211)

"Mais comment trouver son chemin au fond de ces fumées et ces miroirs" (p. 49), se demande Johnny? Comment avancer si l'on ne voit rien ou si l'on ne voit que soi-même? Le roman ressemble à ce chemin hivernal qu'il décrit: "Parlez-moi d'un voyage blanc entre deux berges blanches." (p. 281) Le cahier de Walter est une longue impasse que Johnny s'obstine à suivre jusqu'au bout, incapable de se résoudre à rebrousser chemin. Il ne s'en débarrassera qu'à la toute fin du roman - comme par hasard le plus long que Ducharme ait écrit en lançant le cahier dans les broussailles où il l'avait d'abord trouvé.

Tout est circulaire dans Gros mots, le récit comme l'île dont Johnny ne cesse de faire le tour. Même lorsqu'il rend visite à Petite Tare, il annonce qu'il s'en va faire le "tour d'Elle". L'Alter Ego est une figure du Même, tout comme Julien, le frère de Johnny, est un "fou de l'identité " (p. 14). Rien ne change, rien n'évolue, mais attention: cette circularité a ses exigences et, pour y pénétrer, il faut y mettre du sien et croire à certains principes. C'est précisément ce que fait Johnny, dont la nullité même appelle à un maximum de résistance:

\begin{abstract}
Résister. Tout le long en remontant aux Deux-îles, je me le suis répété. La loi qui force à changer, à progresser ou régresser, s'épanouir puis se flétrir, on se cramponnera et on la violera, on ne se soumettra pas. Je veux que ça reste comme c'est, toujours pareil. Il n'y a pas à chercher mieux, il n'y a pas mieux. (p. 157)
\end{abstract}

Ducharme écrivait à peu près la même chose trente ans plus tôt, dans Le nez qui voque, comme s'il obéissait en 1999 à l'injonction de son personnage et s'amusait à se citer luimême, à se mirer dans ses propres textes. Rappelons les termes de Mille Milles:

Restons en arrière avec Crémazie, avec Marie-Victorin, avec Marie de l'Incarnation, avec Félix Leclerc, avec Jacques Cartier, avec 
Iberville et ses frères hérö̈ques. Restons en arrière. Restons où nous sommes. N'avançons pas d'un seul pas. Restons fidèles. Souvenons-nous. Le temps passe: restons ${ }^{6}$

La constance de l'écriture de Ducharme ne fait aucun doute. Le rapprochement des deux textes écrits à trente années de distance fait cependant ressortir une importante nuance qui révèle à quel point, même dans la continuité, l'écriture de Ducharme se renouvelle. Dans le texte de 1967, le verbe "rester" impliquait non seulement un désir de ne pas changer, mais aussi une litanie de héros du passé. Mille Milles disait: "restons en arrière", et s'inventait une famille, un "nous". Johnny, lui, passe du "je" au "on" impersonnel, sans citer le moindre nom propre. Les écrivains qu'il convoque, d'Emily Dickinson à Proust, ne forment pas pour lui une famille, un "nous". Seul ou dédoublé (avec son Alter Ego), il n'appelle plus à rester "en arrière", mais veut obstinément que "ça reste comme c'est, toujours pareil . La régression ne vaut désormais pas mieux que la progression, toutes deux étant renvoyées dos à dos du côté de la loi. L'échec de Johnny-Walter trouve peut-être ici son sens véritable, celui de la plus extrême redondance.

$\mathrm{Au}$ bout de la redondance, il y a toutefois autre chose qui surgit. Bien qu'ils ressemblent à d'autres personnages de Ducharme, Exa Torrent, Julien ou Petite Tare ont quelque chose de moins concrets, de moins immédiatement attachants aussi peutêtre. Les colères d'Exa, la faiblesse de Petite Tare, nous les observons de loin, à travers un prisme déformant qui n'est pas seulement celui du cahier de Walter, mais aussi celui, plus déformant encore, d'un langage romanesque livré à d'inexplicables distorsions. Ce n'est pas nouveau, dira-t-on avec raison. Mais avec Gros mots, c'est pire que jamais. Ce n'est plus seulement une langue (comme le bérénicien) que Ducharme invente, c'est une grammaire. En commentant un mot d'Exa qui contenait une faute d'accord ("Tu as raison, c'est moi qui a commencé" (p. 260), Johnny prend la peine d'expliquer au nom de quelle règle ce n'est pas vraiment une faute: "elle fait accorder le verbe avec le pronom sous-entendu mis en apposition, et elle a bien le droit.... Il ajoute, joignant le geste à la parole: "Et je lui donne raison gratis, par comme elle, qui s'est mise à me donner raison sur tout pour que je lui donne tout le reste, avec ma bénédiction pour aller se faire ligoter et mieux passer à tabac." (p. 260, je souligne) Cette grammaire amoureuse, offerte et appliquée pour donner raison à Exa dans l'un des rares instants de tendresse qu'ils partagent, fait de Gros mots un roman où le lecteur ne cesse de s'accrocher, de trébucher sur le langage. Le romancier s'amuse à changer les règles, à brouiller les noms, à désarticuler la syntaxe au risque de rendre certaines phrases illisibles. Mais qu'est-ce que l'illisibilité de quelques phrases dans un monde de "Gros mots", dans un univers de signes redondants où le même se confond avec l'autre?

Le langage de Gros mots, tordu non pas à l'extrême, mais à des degrés variables (et donc imprévisibles), se situe lui aussi du côté de la résistance, au sens physique du terme cette fois. Le récit en lacets oblige le lecteur à garder le pied sur le frein. Une chronique comme celle- 
ci ne se prête pas à une analyse en profondeur des nombreux effets de manche du romancier, mais ce serait passer à côté du roman que de ne pas insister sur la difficulté de lecture que pose Gros mots. Je prends à peu près au hasard les phrases suivantes (pour bien faire, il aurait fallu montrer que tout le paragraphe reprend symétriquement le paragraphe précédent qui décrit une action similaire effectuée par Walter plutôt que par "Je"):

Je suis sorti plus tôt, j'ai marché sur la glace. Il faut patauger un peu, des fois jusqu'aux cuisses, en franchissant les rouleaux, mais le vent les a formés en déblayant d'impeccables parquets, il vous les a balayées et vous les a polies, vous pourriez vous y mirer. (p. 247)

Avec quels mots s'accordent les participes passés "balayées" et "polies"? La logique voudrait que ce soit avec "parquets", mais d'où vient alors le féminin? N'insistons pas... Plus loin, la phrase, moins grandiose que la précédente, mais plus musicale encore, contient aussi un solécisme qui fait achopper la lecture: "Loin des maisons, c'est beau comme un ailleurs, on est seul au monde et ravis, avivés, passionnés de respirer..." Pourquoi "seul" au singulier et "ravis ", "avivés " et "passionnés" au pluriel? Continuons. "Ça me rappelait, sous un jour idéal, le temps où nous traversions, droit devant nous, prendre un apéritif au Point du Soir. "Cette fois, rien ne cloche: la licence syntaxique courante (l'ellipse de la préposition "pour" devant "prendre") permet à Ducharme de rapprocher les deux actions, les deux temps, c'est-à-dire la traversée et le résultat de la traversée. Puis retour en arrière, à la faveur duquel l'avant, le pendant et l'après se mêlent et se répètent à l'infini, par l'intermédiaire de ce souvenir qui peut paraitre secondaire, mais qui conduit au thème central du roman, à savoir la résistance à la disparition de soi : "On s'était battu un sentier, un chapelet de foulées qu'après les tempêtes on s'amusait à recréer, suivant les ombres en creux laissées par nos traces effacées." (p. 247) Le verbe pronominal "se battre", détourné de son usage habituel (il devient ici transitif), suggère bien qu'il s'agit d'un combat dans le langage luimême, un combat toujours à recommencer. Contre quoi? Contre ce qui efface les traces. Pour guider les pas (les "foulées") dans ce paysage hivernal, trop blanc, le sujet menacé d'anéantissement n'a que les "ombres en creux laissées par nos traces effacées".

Prenons un autre exemple pour bien montrer l'ampleur nouvelle de la catastrophe. Dans L'biver de force, les deux héros, Nicole et André, voulaient en finir avec la réalité, celle des choses comme celle des mots: leur principal, leur seul projet en fait, consistait à faire en sorte qu'il n'y ait plus rien. Ils s'acharnaient par conséquent à vider les lieux, les leurs et ceux des autres, ceux du discours qu'on appelle les lieux communs. Ils vident leur appartement et se retrouvent, exaltés, tout nus dans la neige, devant un vide riche en quelque sorte du geste qui le créait. Tel était le sens de leur action: "On est un vide qui se refait, que c'est ça notre sens, et on est contents ${ }^{7}$. Johnny et Petite Tare, eux, ne commettent aucune action de cet ordre pour détruire la réalité, pour quitter le 
"jumbo-bateau 8 " sur lequel avance ce qu'on appelle la société. La seule action qu'ils posent consiste à préserver de la destruction leur amour, menacé de sombrer dans le vide où tout s'abîme autour d'eux:

Vivre d'amour. Vivre d'aimer ma Petite Tare. Ne faire que ça. Tout laisser périr, s'engouffrer dans le vide en train de se faire, excepté ça, qui s'y répandra infiniment, l'envahira comme un ciel bleu sans soleil, éclairé de l'intérieur. C'est tout simple, il suffit d'y penser, tout le temps. (p. 231)

On reconnaît encore une fois la propension de Ducharme à s'autoplagier, à travers l'allusion à un "vide en train de se faire", expression empruntée presque telle quelle à L'biver de force. En 1973, toutefois, le vide tendait à se combler de lui-même et il fallait sans cesse le re-faire. En 1999 , le vide se fait tout seul, emportant tout avec lui, même le héros qui sent bien, dès l'ouverture du roman, qu'on cherche à se débarrasser. de lui. D'où le privilège accordé dans Gros mots au verbe "résister ", qui renvoie moins à une action commune qu'au salut de l'individu, menacé de sombrer corps et biens dans le vide. Cet individu ne cherche pas à promouvoir l'individualisme: les signes extérieurs de son existence ne l'intéressent pas. Tout ce qui compte, pour lui, c'est ça, ce qui veut dire, dans les termes que Ducharme reprend à une époque qui paraît révolue: "Vivre d'amour. Vivre d'aimer ma Petite Tare."

Et Exa? À en juger par la grandeur des disputes qu'ils s'offrent, Exa fait partie du ça de Johnny. Il a besoin des deux, des trois, faudrait-il dire en incluant Poppée, la danseuse topless qu'il parvient à séduire.
Toutes ces figures, la sœur, la femme et la putain, ne sont jamais dissociables. Non seulement Johnny va et vient de l'une à l'autre, mais il les fond dans la figure supérieure de Petite Tare: "Tu es notre petite pute et notre petite sœur" (p. 229), lui ditil. C'est elle qui l'avait supplié, non sans perversion, d'avoir une aventure avec Poppée. Pourtant, Petite Tare aime Johnny "De très haut... Sans toucher chair... Le grand amour abstinent unanime, quoi." (p. 229) Lorsque Exa quitte le domicile conjugal et s'en va rejoindre un de ses amants, Petite Tare offre à Johnny le double de ses clefs, de toutes ses clefs. Il n'en demandait pas tant, lui qui n'aime que ce qui résiste. Les portes s'ouvrent toutes grandes, trop grandes: Exa le quitte pour de bon, le mystère du cahier de Walter se dissipe (grâce à la remplaçante de Poppée, qui l'avait connu) et, à la toute fin, Petite Tare part avec un musicien. Il ne reste plus à Johnny qu'à trouver un travail et à disparaître, lui aussi, à se perdre dans la société. "Moi obstacle aboli" (p. 309), conclut-il, retirant de la scène, sans tragédie, comme toujours pour ainsi dire, ce moi qui n'était plus un obstacle pour personne.

1. Réjean Ducharme, Gros mots, Paris, Gallimard, 1999, $311 \mathrm{p}$.

2. Id., Le nez qui voque, Paris, Gallimard, 1967, p. 89.

3. "Je ne suis pas un homme de lettres. Je suis un homme", lit-on en préface au Nez qui voque (op. cit., p. 8).

4. Voir les cahiers du Nez qui voque et de L'biver de force qui se trouvent aux Archives nationales à Ottawa (fonds Réjean-Ducharme).

5. "Je marche à côté de moi en joie / J'entends mon pas en joie qui marche à côté de moi " ( Accompagnement ", Euvres, édition critique par Jacques Brault et Benoît Lacroix, Montréal, Presses 
de l'Université de Montréal, coll. "Bibliothèque des lettres québécoises *, 1971, p. 34). Plus loin, Ducharme nomme et cite (presque) littéralement deux vers de Saint-Denys Garneau (op. cit., p. 285).
6. Le nez qui voque, op. cit., p. 29

7. Réjean Ducharme, L'biver de force, Paris, Gallimard, 1973, p. 181 ; c'est l'auteur qui souligne.

8. Ibid., p. 13. 